\title{
ACCESSIBILITY TO BREAST CANCER TREATMENT AND BARRIERS TO HEALTH ASSISTANCE
}

Júlia J. Caetano', Daniel B. Sousa1, Leonora S. F. Couto', Paula O. C. Queiroz¹, Bruna E. Miranda

${ }^{1}$ Faculdade de Medicina, Universidade Federal de Goiás - Goiânia (GO), Brazil.

Objective: Despite developed countries having the highest incidence rates of breast cancer (BC), mortality rates are higher in developing countries, due to late diagnosis and difficulties to accessing care. This study aimed to evaluate the access to $\mathrm{BC}$ treatment in Brazil. Methodology: This is a narrative, exploratory, and qualitative review of the accessibility to BC treatment. The search for articles happened on platforms SciELO and Scholar. The period of publications was from 2009 to 2019. The descriptors were: accessibility, treatment, and breast cancer. Results: Accessibility is essential in the prevention and treatment of BC, but this is not the current Brazilian reality. Since 2011, 20,828 surgeries and 37,000 hospitalizations were performed by Sistema Único de Saúde. However, almost $40 \%$ of the total is concentrated in seven capitals, São Paulo, Rio de Janeiro, Belo Horizonte, Porto Alegre, Curitiba, Salvador, and Fortaleza. Some capitals, such as Recife, Porto Alegre, Belo Horizonte, and Curitiba, showed a predominance of non-resident attendances, respectively, of 71.4, 60, 53.7, and 50.7\%. The mean distance traveled was $67 \mathrm{~km}$, evidencing a geographical barrier making treatment continuity difficult. In a survey of barriers in health care to BC, conducted with 58 women, treatment period was referred to as the main obstacle, totaling 23. Then, a total of 22 reported organizational barriers, including the waiting list for consultation, exams and treatments, and the error of diagnosis. In their view, those responsible for these events were the hospital and the Municipal Secretaries or Health System. Finally, the lack of information about the disease and economic barriers were reported. Conclusion: It is necessary to prioritize public policies that guarantee access to early diagnosis and treatment. Such measures should increase the survival and guarantee smaller losses in the quality of life. In addition, they can reduce more expensive treatments resulting from tumor progression. 